\title{
Erratum to: Effect of Bolus Viscosity on the Safety and Efficacy of Swallowing and the Kinematics of the Swallow Response in Patients with Oropharyngeal Dysphagia: White Paper by the European Society for Swallowing Disorders (ESSD)
}

\author{
Roger Newman $^{1,5} \cdot$ Natàlia Vilardell $^{2,5} \cdot$ Pere Clavé $^{1,2,3,5} \cdot$ Renée Speyer $^{1,4,5}$
}

Published online: 21 July 2016

(C) Springer Science+Business Media New York 2016

Erratum to: Dysphagia (2016) 31:232-249

DOI 10.1007/s00455-016-9696-8

For this article, Open Access was ordered after publication.

This requires a change of the copyright and license terms.
This article is distributed under the terms of the Creative Commons Attribution 4.0 International License (http:// creativecommons.org/licenses/by/4.0/), which permits unrestricted use, distribution, and reproduction in any medium, provided you give appropriate credit to the original author(s) and the source, provide a link to the Creative Commons license, and indicate if changes were made.

The online version of the original article can be found under doi:10.1007/s00455-016-9696-8.

Pere Clavé

executiveofficer@myessd.org

1 College of Healthcare Sciences, James Cook University, Townsville, QLD, Australia

2 Unitat d'Exploracions Funcionals Digestives, Department of Surgery, Hospital de Mataró, Universitat Autònoma de Barcelona, Mataró, Spain

3 Centro de Investigación Biomédica en Red de enfermedades hepáticas y digestivas (CIBERehd), Instituto de Salud Carlos III, Barcelona, Spain

4 Leiden University Medical Centre, Leiden, The Netherlands

5 European Society for Swallowing Disorders (ESSD), Carretera de Cirera s/n, 08304 Mataró, Spain 